\title{
Multipoint temperature-independent fiber-Bragg-grating strain-sensing system employing an optical-power-detection scheme
}

\author{
Yan-Ju Chiang, Likarn Wang, Horng-Shyang Chen, Chih-Chung Yang, and Wen-Fung Liu
}

\begin{abstract}
A temperature-independent fiber-Bragg-grating strain-sensing system, based on a novel optical-powerdetection scheme, is developed and analyzed. In this system a pair of fiber Bragg gratings with reflection spectra either partially or substantially overlapping is placed side by side to form a temperatureindependent strain-sensor unit. Conventional wavelength-interrogation techniques are not used here, and instead an optical-power-detection scheme is proposed to directly calibrate the measurand, i.e., the strain. Unlike the conventional approach in a multiplexed sensing system, the presented powerdetection-based interrogation method does not need the fiber-Bragg-grating sensors to be spectrally separate. The only requirement is that the spectra of the two fiber Bragg gratings of each sensor unit in a multiplexed system be identical or slightly separate (slightly overlapping spectra would also work in the sensing scheme) and the source's optical power be sufficient for sensitive measurement. Based on a three-sensor-unit system, we demonstrate simple strain measurements of high linearity $( \pm 0.4 \%)$, good sensitivity $[2$ microstrains $(\mu \mathrm{S})]$, high thermal stability $( \pm 0.8 \%)$, and zero cross talk. The effects of light source spectral flatness and fiber bending loss on measurement accuracy are also discussed. (C) 2002 Optical Society of America

OCIS codes: $\quad 060.2310,060.2370,050.2770,040.1880,120.6810,060.2410$.
\end{abstract}

\section{Introduction}

Intracore fiber Bragg gratings (FBGs) have become attractive optical components for sensing because of their compactness and wavelength-encoding capability. When the FBGs are applied as sensors, the measurands can be temperature, strain, pressure, magnetic field, and others. ${ }^{1,2}$ These measurands are determined by detecting the wavelength shift (i.e., the Bragg wavelength shift) of the light backreflected from the FBGs. In either a single-point sensor or a multiplexed sensor system, the Bragg wavelength shift can be measured by employing a wavelength interrogation scheme with a tunable fil-

Y.-J. Chiang and L. Wang (lkwang@ee.nthu.edu.tw) are with the Department of Electrical Engineering, National Tsing Hua University, Hsinchu, Taiwan 300. H.-S. Chen and C.-C. Yang are with the Department of Electrical Engineering and the Graduate Institute of Electro-Optical Engineering, National Taiwan University, Taipei, Taiwan. W.-F. Liu is with the Department of Electrical Engineering, National Defense University, Tahsi, Taoyuan, Taiwan.

Received 22 June 2001; revised manuscript received 10 October 2001.

0003-6935/02/091661-07\$15.00/0

(C) 2002 Optical Society of America ter, ${ }^{3}$ an interferometer, ${ }^{4}$ a diffraction grating and CCD spectrometer, ${ }^{5}$ a frequency-locking circuit, ${ }^{6}$ or a frequency-modulated multimode laser. ${ }^{7}$

Strain measurement with FBGs has been an important subject in applications associated with monitoring stressed composite materials. A sensor system with an array of wavelength-multiplexed FBGs has been widely proposed for strain measurement in a smart structure. ${ }^{8-11}$ Many FBG strainsensor systems have been further developed to operate in a temperature-independent mode. For an ac strain measurement, use of a single FBG in conjunction with a local locking circuit can suffice to discriminate the ac strain response from the slowly varying temperature response. However, it is not possible to do so with only one FBG used for measuring a static or quasi-static strain. Various methods have been proposed to resolve this situation. A frequently used method is to utilize a pair of FBGs (written into the same location to form a superstructure) that exhibits different sensitivities to temperature and strain. ${ }^{12,13}$ Dual FBGs could also be structured to form a cavity for simultaneous measurement of strain and temperature. ${ }^{14}$ These methods require calibration for the two sensitivities of each FBG in advance, resulting in some complexity in 
application. However, note that some temperatureindependent strain measurements have been carried out without such calibration by simply nullifying the strain sensitivity of one FBG while keeping the same temperature sensitivity for both FBGs. A recent case of temperature-independent strain sensing exemplified this by employing a chirped FBG partially embedded in a glass tube to obtain a direct opticalpower-detection scheme. ${ }^{15}$ Although simple in structure, this detection scheme has not yet provided a solution to linear and accurate strain measurement.

In this study we propose an alternative to temperature-independent strain measurement by using an optical-power-detection scheme. Quite different from the scheme used in Ref. 15, the proposed detection scheme can not only linearly and accurately measure the strain by using a pair of uniform FBGs but also make a good strain-measurement sensitivity attainable. Although a similar power-detection method was used in Ref. 11, there was no temperature-compensating scheme employed in that research. Another feature that makes the research here different from that in Ref. 11 is that in a multisensor system the presented interrogation method does not need spectrally separate fiber Bragg gratings. In this paper we present this feature by demonstrating strain measurement for a three-pointsensor system. It is organized as follows. In Section 2 we describe the principle of proposed temperature-independent strain sensing. In Section 3 we give experimental results. We discuss the practicability of the proposed optical-power-detection scheme in terms of its linearity, sensitivity, and, more important, unsusceptibility to fiber bend loss. Then in Section 4 we conclude this paper.

\section{Principle of Measurement}

The proposed optical-power-detection scheme for temperature-independent strain measurement is explained with an example of a single-point sensor, as shown in Fig. 1. The sensor unit comprises two FBGs written separately on two different fibers. It is required in the scheme that the reflection spectra of these FBGs be either identical or slightly overlapping, and that the pair of FBGs be structured so that only one is insusceptible to strain effect while both have the same temperature sensitivity. The latter requirement can be realized by embedding FBG 1 in a thin glass tube (sealed with glue at both ends) and keeping FBG 2 free but placing it adjacent to the tubed FBG 1. Note that FBG 2 can also be protected with a tube and left free in the tube experiencing extension/contraction when the fiber line outside the tube is under axial stress. This design is particularly important when the sensor is embedded in a stressed material to insure that both FBGs have an identical thermal effect but experience different strain effects. To explain the principle of operation of the sensing scheme, we assume that the reflection spectra of both FBGs are almost identical both in shape and in central wavelength (although slightly

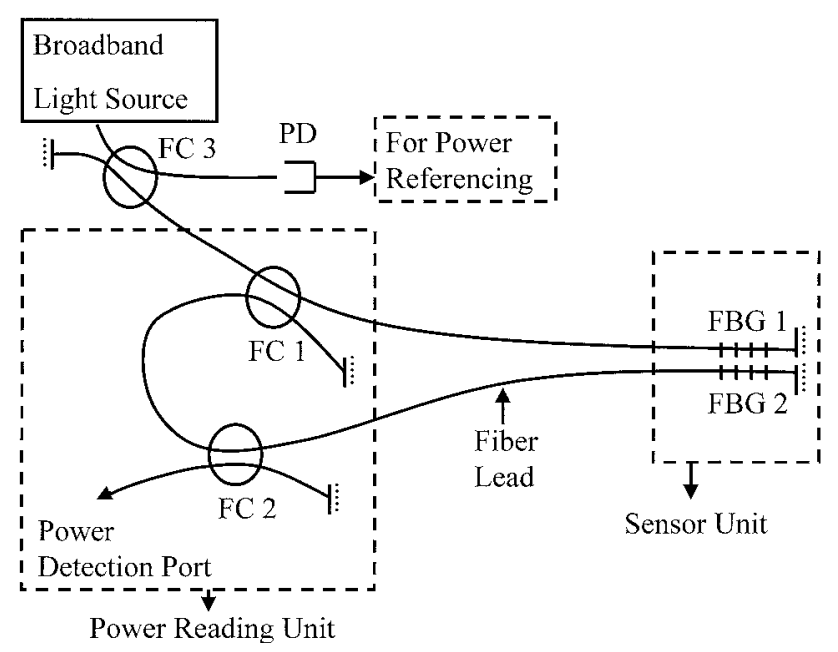

Fig. 1. Proposed power-detection scheme for temperatureindependent strain measurement: FC, fiber coupler; FBG, fiber Bragg grating. The fiber coupler, FC 3 , is used to tap out part of the input power for the purpose of power referencing. All unused fiber ends are immersed in index-matching fluids.

overlapping spectra can work as well). When a broadband light reaches FBG 1 the light at its reflection band is backreflected and directed to FBG 2 through fiber couplers 1 and 2 (i.e., FC 1 and FC 2 of Fig. 1). Now only the part of light that corresponds to the intersection between the spectra of the two FBGs is backreflected by FBG 2 and finally goes through FC 2 and is detected by an optical power meter/detector.

Thus the intersection of the reflection spectra of the two FBGs can be calibrated by the optical power measured at the power-detection port. Because FBG 2 experiences a strain effect (while FBG 1 does not in the designed tubing structure), its reflection spectrum shifts in accordance with the strain. This induces a change in the intersection of the two reflection spectra and accordingly a variation in the detected optical power. Because both FBGs have an identical thermal effect in the design the spectral shift from temperature influence is canceled, while only the strain effect is kept. And this leads to a temperature-independent strain-sensing scheme. To avoid Fresnel reflections that would be superimposed onto the useful signal, antireflection coatings or beveled facets are needed at the extremities of the fibers. In the experiments here, however, indexmatching fluids were used to have the same function. To see how this sensor system would result in a linear strain measurement, we calculate the optical power that would be detected at the power-detection port as a function of strain. Here we assume a strain sensitivity of $1.21 \mathrm{pm} /$ microstrain $(\mu \mathrm{S})$ for FBG 2 . The reflection spectra of both FBGs are assumed to be in super-Gaussian shape, as shown in Fig. 2(a). Here we have also assumed a spectral width of $1.5 \mathrm{~nm}$ for both FBGs and two spectra that are slightly separate (see the solid and dashed curves). With such reflection spectra the optical power detected at the detec- 

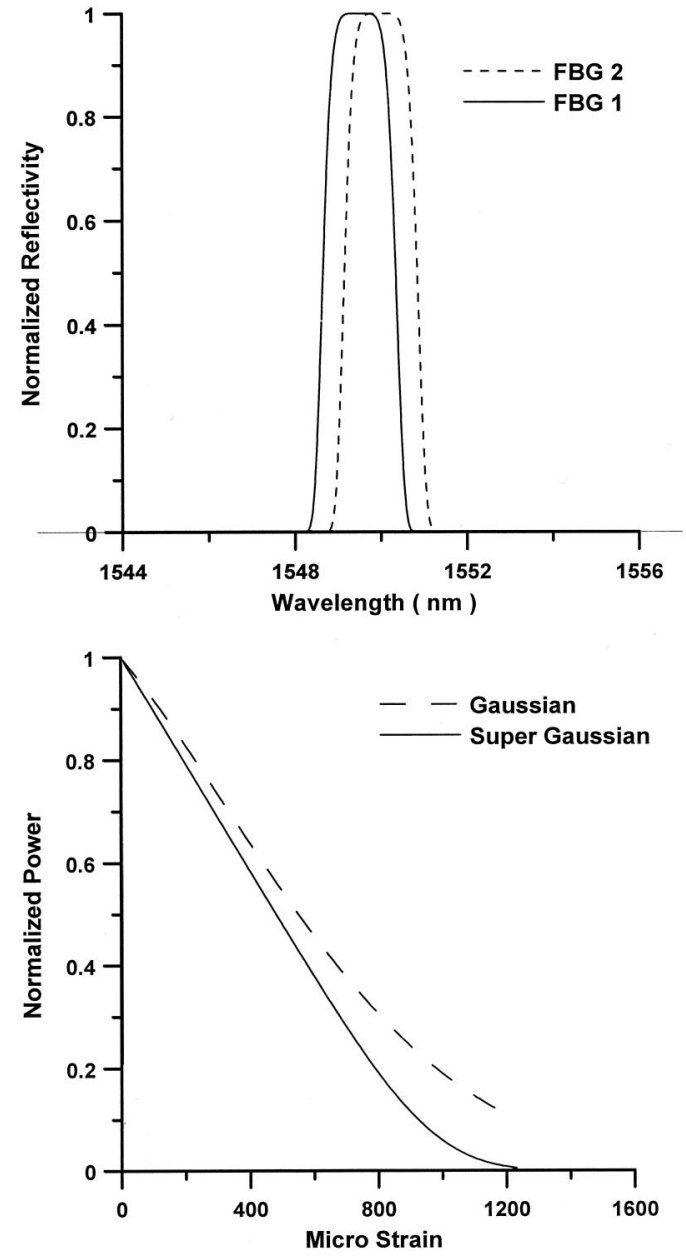

Fig. 2. Simulated result of the strain measurement: tion spectra of both FBGs in super-Gaussian shape. lated detected optical power as a function of strain. Here both spectra are assumed to be $1.5 \mathrm{~nm}$ wide. A strain sensitivity of $0.001 \mathrm{~nm} / \mu \mathrm{S}$ for FBG 2 is assumed. (b) Results for the case of Gaussian-shaped spectra for comparison are also shown.

tion port would then vary with respect to a strain applied to FBG 2, as illustrated by the solid curve in Fig. 2(b). Here quite linear behavior can be observed in the range from 0 to more than $900 \mu \mathrm{S}$. The dashed curve in Fig. 2(b) describes the case in which Gaussian-shaped spectra are assumed for the two FBGs. It shows the slight measurement nonlinearity caused by using FBGs with reflection spectra that are not similar to square-shaped ones. The fiber coupler near the source end (i.e., FC 3), its function being explained below, is also shown in Fig. 1 although not used in the experiment here.

The power-detection scheme in Fig. 1 can apply to multipoint sensing as illustrated in Fig. 3, where an optical power splitter is used to distribute broadband light into each sensor unit. The broken-line frames in Fig. 3 represent the power reading unit of Fig. 1. Because each sensor unit operates independently in a power-detection mode, the Bragg wavelengths of different sensor units can be the same. The requirement for obtaining sensitive strain measurement is

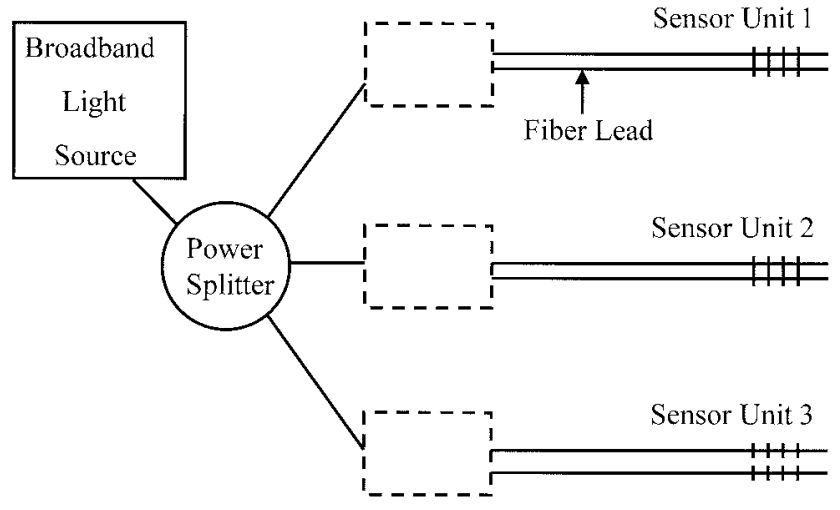

Fig. 3. Multipoint strain-sensing system using the powerdetection scheme. The broken-line frames represent the power reading unit of Fig. 1.

that sufficient optical power be supplied by the broadband source. The measurement sensitivity is dependent on the power of the source fed into the sensor unit against the noise level of the electronic circuit in optical power detection.

\section{Experimental Results and Discussion}

We have carried out a set of experiments for threepoint strain measurements. The amplified spontaneous emission noise from a section of $\mathrm{Er}^{3+}$-doped fiber pumped by a $980-\mathrm{nm}$ diode laser is used as the broadband light source. A 30:70 fiber coupler and 50:50 fiber coupler form the optical power splitter required for the presented measurements in which nearly identical amplified spontaneous-emission power is launched into each sensor unit. In each sensor unit the two FBGs used are inserted into thin glass tubes and placed side by side. One of the tubes is sealed at both ends so that the FBG in it is kept slightly loose and would not experience an axial strain effect. The other FBG is left free in its tube so that an axial strain would induce a Bragg wavelength shift on it. In our experiments the glass tubes have nearly the same inner diameter of $\sim 0.5 \mathrm{~mm}$ and the outer diameter of $\sim 1.5 \mathrm{~mm}$. The center-to-center separation of the tubes is smaller than $2 \mathrm{~mm}$. In such an arrangement it is assumed that both FBGs in each sensor unit are in the same thermal condition, and thus the strain measurement would be temperature insensitive as explained above.

The reflection spectra of the FBGs used for the three sensor units in the condition of zero strain are shown in Fig. 4. Obviously the pair of FBGs for each of the sensor units 1 and 2 has substantially overlapping reflection spectra [see Figs. 4(a) and 4(b)], whereas the reflection spectra of the pair of FBGs for sensor unit 3 only slightly overlap [see Fig. 4(c)]. Note that the reflection spectra of the FBGs for each sensor unit are not well separated from those for any other sensor units. The reflection spectra (or Bragg wavelengths) of the FBGs for sensor unit 2 are even essentially in the same band as those for sensor unit 3. This situation features the proposed 


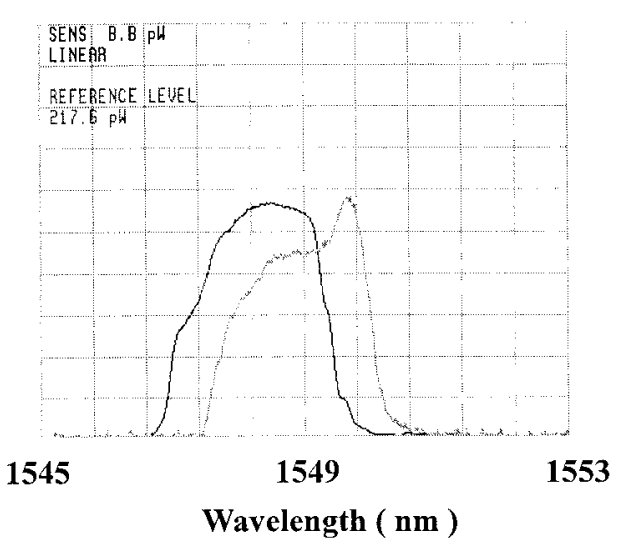

(a)

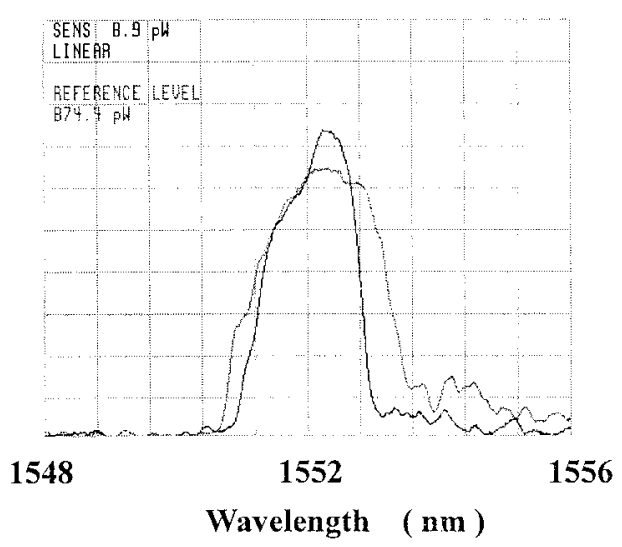

( b )

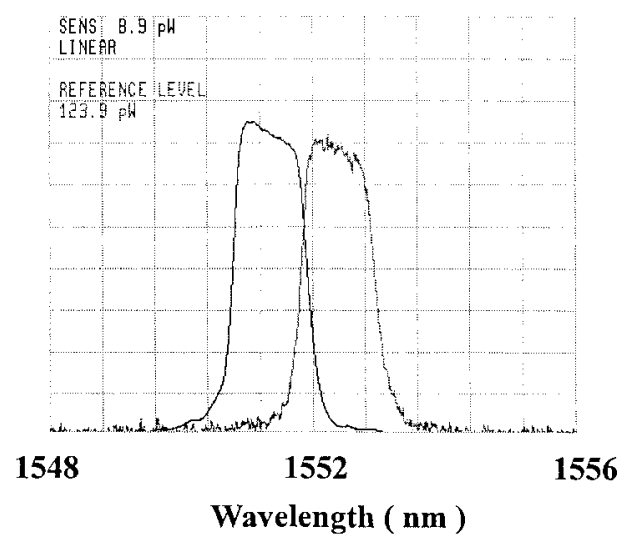

(c)

Fig. 4. Normalized individual reflection spectra of (a) sensor unit 1 , (b) sensor unit 2 , and (c) sensor unit 3.

power-detection-based multipoint strain-sensor system as opposed to the conventional wavelengthinterrogation approaches in which the Bragg wavelength of each Bragg grating must be separated far enough from the others.
In practical application only one FBG of each sensor unit of the proposed system can experience extension/contraction (which induces a Bragg wavelength shift) when the fiber line outside the tube is under axial stress. In our experiments the loosely tubed FBG of each sensor unit is pulled with a translation stage to produce an axial strain effect. In this way we apply first a strain on sensor 1 only (i.e., in the meantime keeping other sensor units free of any axial strain) and then measure the optical power at the power-detection port. The solid squares in Fig. 5(a) show the detected optical power as a function of axial strain. Quite linear behavior can be seen for axial strain as great as $\sim 1400 \mu \mathrm{S}$. The linearity is observed to be $\pm 0.38 \%$ in the range of $0-970 \mu \mathrm{S}$. The electronic noise associated with the power detection sets an upper limit for the measurement sensitivity. In all our experiments the power meter Q82216 Advantest is used, offering a noise level of $-77 \mathrm{dBm}$. For most data the electronic noise level corresponds to a measurement sensitivity of $2 \mu \mathrm{S}$. In Fig. 5(a) we also show the detected optical powers for the other two sensor units (see the solid circles for sensor unit 2 and triangles for sensor unit 3), which are not under axial strain. These powers maintain at constant levels against the axial strain that is applied only to sensor unit 1 , indicating no cross-talk effect in measurement. Figure 5(b) shows the measured optical powers at the detection ports of the three sensor units when the axial strain is applied only to sensor unit 2 . Here the solid circles represent the measured optical power at the power-detection port of sensor unit 2 as a function of axial strain. A slightly nonlinear change in optical power for small strain is noted for sensor unit 2. This result is attributed to the relatively larger difference between the spectral shapes of the two associated FBGs at the overlapping part [see Fig. 4(b)].

Nevertheless the measured data in this case still show a linearity of better than $2 \%$ for axial strain as great as $1500 \mu \mathrm{S}$. The outputs of the other two sensor units maintain at constant levels regardless of the strain applied to sensor unit 2. Likewise, when axial strain is applied only to sensor unit 3 , the detected power for sensor unit 3 varies linearly with the strain [see solid triangles in Fig. 5(c)]. Here the axial strain was applied by pulling the FBG that has a shorter Bragg wavelength [see Fig. 4(c)]. The solid squares and circles in Fig. 5(c) are the measured data for sensor units 1 and 2, respectively. Again the constant levels of the data reveal zero cross talk in measurement.

In another set of experiments we measure the thermal response of each sensor unit to ensure its temperature insensitivity in strain sensing. First, we show in Fig. 6 three spectra measured at the detection port of sensor unit 1 when the sensor unit is at $22{ }^{\circ} \mathrm{C}$ (left), $50{ }^{\circ} \mathrm{C}$ (central), and $80{ }^{\circ} \mathrm{C}$ (right) under zero axial strain. Little change in the spectrum shape can be seen as the temperature varies. Similar results were observed for the other two sensor units. Thus the detected optical power is expected 


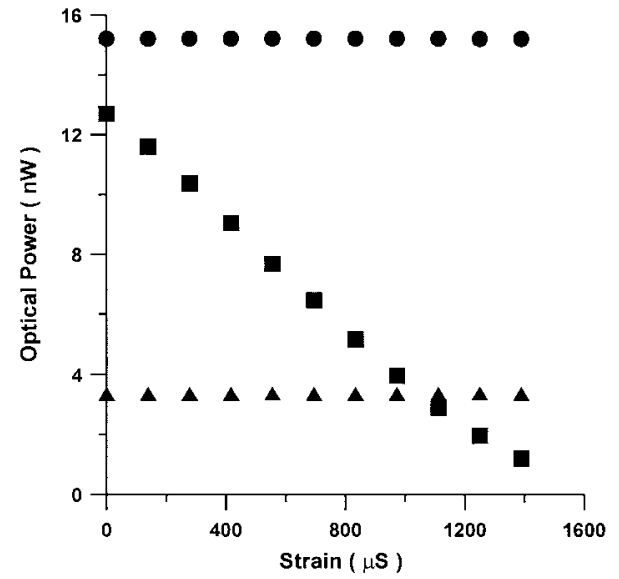

(a)

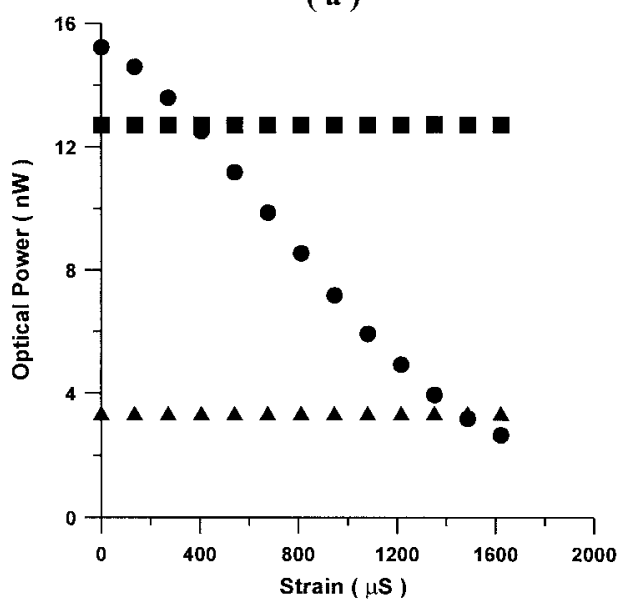

(b)

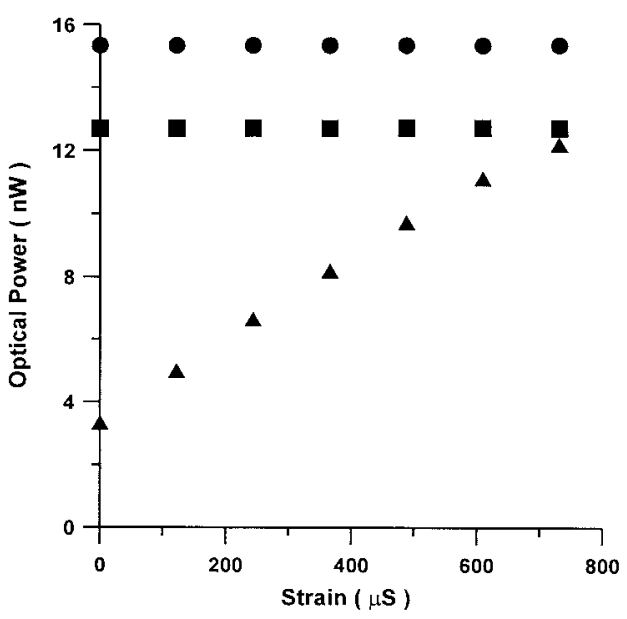

(c)

Fig. 5. Detected optical power versus the axial strain applied to (a) sensor unit 1, (b) sensor unit 2, and (c) sensor unit 3, while the other two are kept strain free. Solid squares, circles, and triangles represent the data points of sensor units 1,2 , and 3 , respectively.

to be independent of the thermal condition. Figure 7 shows the measured optical power at the detection port of each sensor unit (in the condition of zero axial strain) as a function of temperature when each sensor unit is individually heated. The solid squares, solid

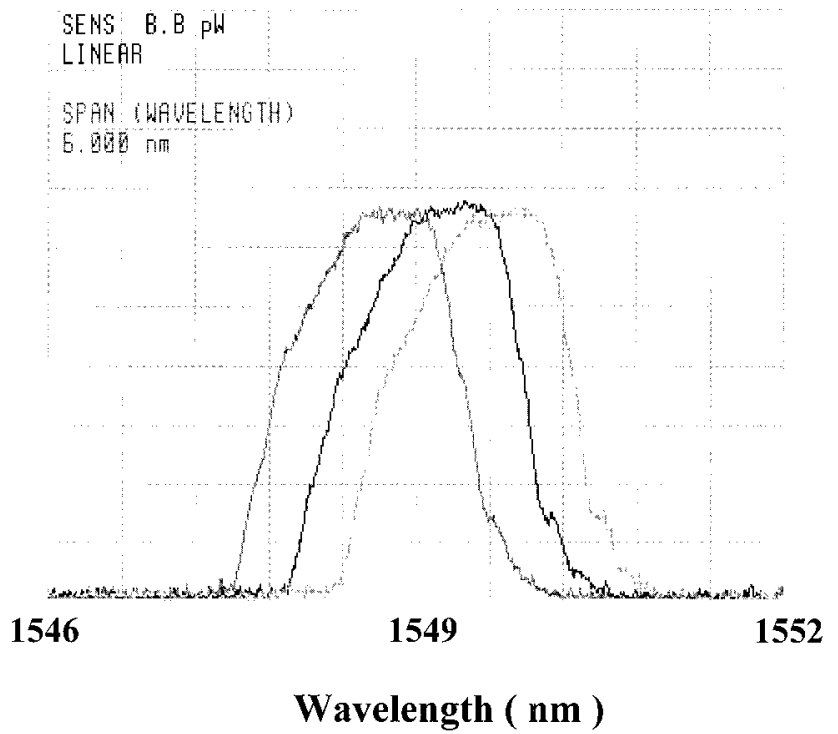

Fig. 6. Three overlapping spectra measured at the detection port of sensor unit 1 when the sensor unit is kept at (left) $22^{\circ} \mathrm{C}$, (middle) $50{ }^{\circ} \mathrm{C}$, and (right) $80^{\circ} \mathrm{C}$, in the condition of zero axial strain.

circles, and solid triangles are the data for sensor units 1,2 , and 3 , respectively. The thermally induced optical power variation for each sensor unit is found to be within $\pm 0.8 \%$ with respect to the individual average value. The slight variation in detected optical power with respect to temperature is attributed to the nonflatness of the source spectrum used in the experiments.

The two curves in Fig. 8 represent the source spectra over the wavelength band of interest. The source spectrum labeled $80 \mathrm{~mA}$ (which represents an injection current to the 980 -nm pump diode) has a relatively larger spectral slope. If this spectrum is used, a larger variation in optical power with temperature is expected. This is because the shapes of the reflection spectra (henceforth the intersection of them) of the pair of FBGs in each sensor unit would

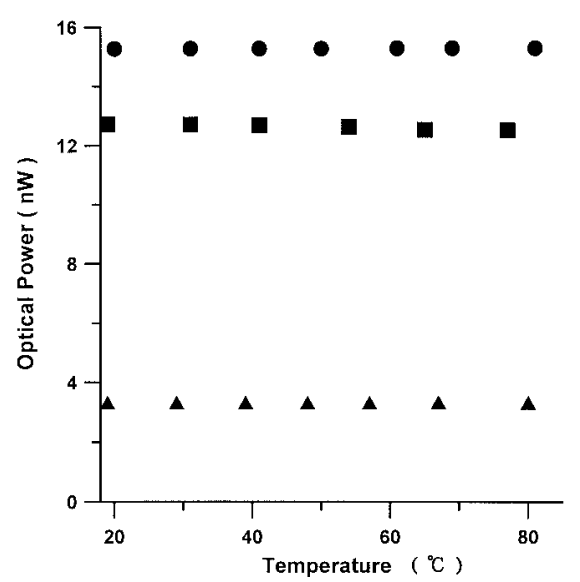

Fig. 7. Optical powers measured at the power-detection ports of solid squares, sensor unit 1 ; solid squares, sensor unit 2 ; and, solid triangles, sensor unit 3 , as a function of temperature when each sensor unit is heated alone. 


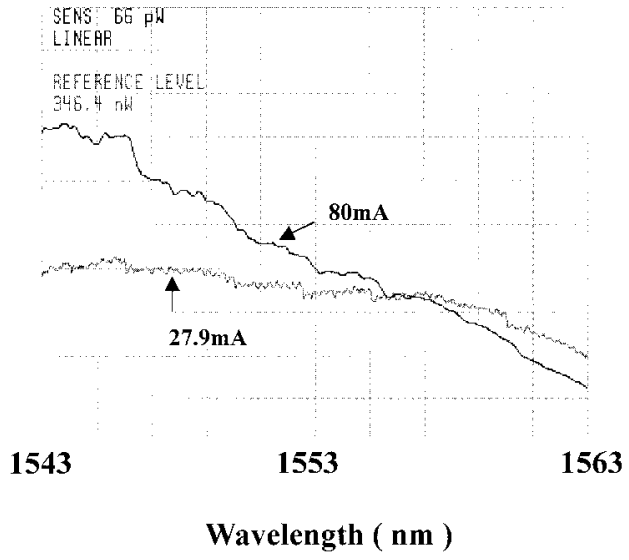

Fig. 8. Spectra of the broadband light source with injection currents of 27.9 and $80 \mathrm{~mA}$.

change as the Bragg wavelengths red shift with increasing temperature. When the shape of the intersection of the two reflection spectra changes, the detected power, which corresponds to the intersection, varies. This problem can be eased by reducing the injection current to obtain a more flattened source spectrum, such as that labeled with an injection current of $27.9 \mathrm{~mA}$ in Fig. 8. All the results described above were obtained in this condition. This is nearly the optimum source spectrum we could have for the measurements here. Generally speaking, a lower injection current results in a more flattened source spectrum at the expense of available optical power.

Then we address an important issue regarding the susceptibility of the proposed sensing scheme to fiber bend loss. As noted the proposed strain sensing is based on detection of the optical power flowing through a long fiber lead. Any unexpected and unpredictable power loss would be a problem for obtaining a reliable measurement. One major cause for such power loss might be the bending of fiber lines. We have tested the proposed sensing scheme by measuring the optical power at a power detection port while intentionally bending a section of the corresponding fiber lead. Figure 9 shows the measured optical power as a function of the radius of curvature of the bend. As can be seen from Fig. 9 the power decrease with a radius of curvature larger than 1.6 $\mathrm{cm}$ is negligibly small $(<0.8 \%)$. This result assures one that power loss due to a macroscopic fiber bend may not be a problem in applying the proposed sensor, because in general a fiber line would not curve so much. It is also important that dynamic microscopic bends with radii of curvature approximating the fiber radius are not produced in the measurement. It might be expected that the effect of microbending can be substantially reduced by suitably coating the fiber lead. The variations of the optical source power and the connector loss (particularly at the source-fiber joint) can present a problem for long-term application in the power-detection scheme, because such variations may be falsely interpreted as a strain change. To resolve this problem, a detector can be used to

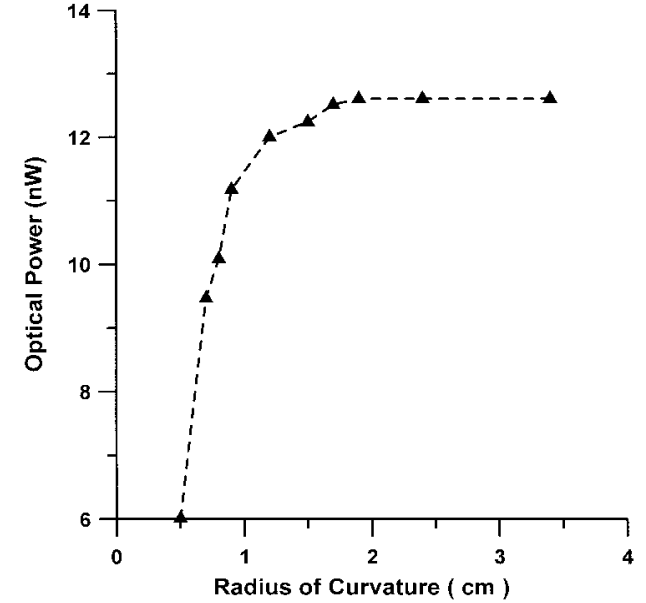

Fig. 9. Measured optical power versus radius of curvature of the bend of a section of fiber lead.

monitor dynamically the optical power entering the sensing scheme for power referencing (see Fig. 1 where FC 3 is used to tap out part of the input power). Similar power referencing is also addressed in Ref. 11.

\section{Conclusion}

We have proposed a method based on optical power detection for multipoint temperature-independent strain sensing. In this method, two uniform FBGs placed side by side in each sensor unit were used to reflect optical power that varied with the measurand, i.e., strain. By simply calibrating the detected power, a strain measurement of high linearity $( \pm 0.4 \%)$, good sensitivity $(2 \mu \mathrm{S})$, and high thermal stability $( \pm 0.8 \%)$ was achieved. The system can be further improved by using a light source of a flat spectrum (for even better thermal stability) and FBGs of large and further squarelike Bragg windows (for even higher linearity and a larger dynamic range). We can further enhance the measurement sensitivity by using a light source of larger power or a more sensitive power detector for each sensor unit. The experimental results also show that no cross talk can be incurred among the sensor units.

This research was sponsored by the National Science Council, The Republic of China, under grants NSC 89-2218-E-002-094 and NSC 89-2218-E-002095 .

\section{References}

1. A. D. Kersey, M. A. Davis, H. J. Patrick, M. LeBlanc, K. P. Koo, C. G. Askins, M. A. Putnam, and E. J. Friebele, "Fiber grating sensor," J. Lightwave Technol. 15, 1442-1463 (1997).

2. J. Mora, A. Diez, J. L. Cruz, and M. V. Andres, "A magnetostrictive sensor interrogated by fiber gratings for dc current and temperature discrimination," IEEE Photon. Technol. 12, 1680-1682 (2000).

3. G. A. Johnson, M. D. Todd, B. L. Althouse, and C. C. Chang, "Fiber Bragg grating interrogation and multiplexing with a $3 \times 3$ coupler and a scanning filter," J. Lightwave Technol. 18, 1101-1105 (2000). 
4. A. D. Kersey, T. A. Berkoff, and W. W. Morey, "High-resolution fiber-grating-based strain sensor with interferometric wavelength-shift detection," Electron. Lett. 28, 236-238 (1992).

5. A. Ezbiri, A. Munoz, S. E. Kanellopoulos, and V. A. Handerek, "High resolution fiber Bragg grating sensor demodulation using a diffraction grating spectrometer and CCD detection," in IEE Colloquium on Optical Techniques for Smart Structures and Structural Monitoring, Digest 1997/033 (Institute of Electrical Engineers, London, U.K., 1997).

6. A. Arie, B. Lissak, and M. Tur, "Static fiber-Bragg grating strain sensing using frequency-locked lasers," J. Lightwave Technol. 17, 1849-1854 (1999).

7. L. A. Ferreira, E. V. Diatzikis, J. L. Santos, and F. Farahi, "Frequency-modulated multimode laser diode for fiber Bragg grating sensors," J. Lightwave Technol. 16, 1620-1630 (1998).

8. D. A. Jackson, A. B. Lobo Ribeiro, L. Reekie, and J. L. Archambault, "Simple multiplexing scheme for a fiber-optic grating sensor network," Opt. Lett. 18, 1192-1194 (1993).

9. K. P. Koo and A. D. Kersey, "Bragg grating-based laser sensors systems with interferometric interrogation and wavelength division multiplexing," J. Lightwave Technol. 13, 1243-1249 (1995).

10. M. A. Davis, D. G. Bellemore, M. A. Putnam, and A. D. Kersey,
"Interrogation of 60 fiber Bragg grating sensors with microstrain resolution capability,” Electron. Lett. 32, 1393-1394 (1996).

11. R. W. Fallon, L. Zhang, A. Gloag, and I. Bennion, "Multiplexed identical broadband chirped grating interrogation system for large strain sensing application," IEEE Photon. Technol. Lett. 9, 1616-1618 (1997).

12. M. G. Xu, J. L. Archambault, L. Reekie, and J. P. Dakin, "Discrimination between strain and temperature effects using dual-wavelength fiber grating sensors," Electron. Lett. 30, 1085-1087 (1994).

13. B. O. Guan, H. Y. Tam, X. M. Tao, and X. Y. Dong, "Simultaneous strain and temperature measurement using a superstructure fiber Bragg grating," IEEE Photon. Technol. Lett. 12, 675-677 (2000).

14. W. C. Du, X. M. Tao, and H. Y. Tam, "Fiber Bragg grating cavity sensor for simultaneous measurement of strain and temperature," IEEE Photon. Technol. Lett. 11, 105-107 (1999).

15. S. Kim, J. Kwon, S. Kim, and B. Lee, "Temperatureindependent strain sensor using chirped grating partially embedded in a glass tube," IEEE Photon. Technol. Lett. 12, 678680 (2000). 